will help stabilize the anti-parallel arrangements, although since the dipoles must be buried to make any significant contribution to energy, they only become important once the hydrophobic core of the protein has been formed.

To conclude, both these papers draw our attention to the contribution which charged groups, including the $\alpha$-helical dipole, make to protein stability. The difficulty of assigning an effective dielectric constant, or indeed if a macroscopic dielectric constant is valid for these atom-atom interactions, has yet to be satisfactorily solved and needs urgent attention. More experimental data for electrostatic energies or effective dielectric constant, such as that provided by Rees ${ }^{8}$, would be of enormous value. With highresolution coordinates now available for many proteins, it should be possible to characterize charge interactions in detail.
A more exciting possibility is that charged groups, being on the surface of proteins, will be of general importance for recognition between molecules, including protein-DNA interactions. Preliminary evidence from the cytochrome $c$ peroxidase structure $^{9}$ and the assembly of tobacco mosaic virus subunits ${ }^{10}$ lends support to this hypothesis. Perhaps it is in this context that direct electrostatic interactions may prove most significant.

1. Wada, A. Adv. Biophys. 9, 1 (1976).

2. Finney, J.L., Gellatly, B.J., Golton, I.C. \& Goodfellow, J. Biophys. J. 32, 17 (1980).

3. Wada, A. \& Nakamura, H. Nature 293, 757 (1981)

4. Hol, W.G.J., Halie, L.M. \& Sander, C. Nature 294, 532 (1981)

. Friend, S.H. \& Gurd, F.R.N. Biochemistry 18, 4620(1979).

6. Tanford, C. \& Kirkwood, J.G. J. Am. chem. Soc. 79, 5333 (1957).

7. Friend S.H \& Gurd, F. R N Biochemistry 18, 4612 (1979). 8. Rees, D.C. J. molec. Biol. 141, 323 (1980)

9. Poulos, T.L. \& Kraut, J. J. biol. Chem. 255, 10322 (1980). 9. Poulos, T.L. \& Kraut, J. J. biol. Chem. 255, 10322 (1980). R. \& Klug, A. Nature 276, 326 (1978)

\title{
Cell mediated immunity in theileriosis
}

\section{from F.E.G. Cox}

THERE are strong indications that antibody does not play a major role in the immune responses to parasitic protozoa that inhibit nucleated cells but there is some evidence that antibody-independent cell-mediated mechanisms may play a part in protection and recovery. Such mechanisms may involve lymphocyte mediated cytolysis (Class I reaction), soluble lymphocyte products (Class II reaction) or natural killer (NK) cells but these mechanisms, although well characterised in mice, are difficult to implicate in the parasitic diseases of man and domesticated animals. It is, however, now clear that cell mediated cytolysis plays a major, if not the major, part in the immune response to one of the most important protozoan diseases of cattle, a form of theileriosis known as East Coast Fever.

Theileriosis is caused by several species of Theileria, of which $T$. parva, in Africa, and $T$. annulata, which occurs in North Africa, the Mediterranean area and elsewhere, are the most important. Considerable mortality results, particularly in calves. Theileriosis is a tick-borne disease and cattle become infected when sporozoites are injected by an infected tick. The sporozoites enter lymphocytes in which they grow and undergo nuclear division giving rise first to macroschizonts and later microschizonts, the products of which infect red blood cells and thence ticks once again. While in the lymphocytes the parasites cause the host cells to undergo rapid division transforming them into what are effectively lymphoblastic cell lines. During the stage of macroschizont production there is an accompanying massive destruction of lymphocytes and the infected animal becomes ill and may die.
Theileria sporozoites can also transform lymphocytes in vitro ${ }^{1-2}$ and the availability of such lymphoid cell lines has provided scientists with an ideal opportunity to study cell mediated immune responses against cells of the same genetic type, for uninfected cells can be taken from an animal before infection, transformed and maintained for subsequent study during the course of an infection ${ }^{3}$. Scientists at ILRAD (International Laboratory for Research on Animal Disease, Nairobi) have followed $T$. parva infections in groups of cattle that died from theileriosis, recovered naturally from the disease or were protected by being infected and treated at the same time with a long acting tetracycline $e^{47}$. In unprotected cattle, by day 14 after infection there was evidence of nonspecific cytotoxic activity against both infected cells and unrelated cells of a mouse tumour line ${ }^{5}$ suggesting a mechanism similar to the natural killer (NK) cell phenomenon seem in mice and humans ${ }^{8}$. In immunised cattle, on the other hand, small numbers of macroschizonts were detected during days 15-18 after infection and cytolysis was confined to genetically identical infected cells ${ }^{5-6}$. An analysis of the mechanisms involved implicated thymus derived lymphocytes as the effector cells ${ }^{7}$ and a restriction mechanism whereby effector cells have to recognise both foreign antigens and self antigens simultaneously 6 . This is characteristic of class I cell mediated immune responses such are seen in virally altered cells ${ }^{8}$. Although the details of class I reactions and the nature of restriction in cattle remain to be

F.E.G. Cox is Professor of Zoology at King's College London.

\section{YEARS AGO}

A SPECIAL despatch has been received at $\mathrm{St}$. Petersburg from M. Sullowsky, dated Irkutsk, December 26 (O.S.) 1881, which says: "At 10 o'clock on the morning of August 9 I parted with the William Rodgers, which shaped her course for Herald Island. The clipper Strelok then returned to the Chinese ports. Up to that time the Strelok and William Rodgers had kept company. They were joined in Providence Bay by an American schooner having on board the captain of a whaler which had stranded. This captain narrated that he had seen a boat with dead men on board which had been driven upon Herald Island. The boat also contained, besides other articles, some silver spoons with the name Jeannette engraved on them. In consequence of this narrative the captain of the William Rodgers resolved to proceed to Herald Island with the view of wintering there, and, with the aid of dogs purchased in Kamschatka, sending out his crew in small parties to the various sides of the island and its vicinity to search for the lost explorers."

Admiral Mouchez will give his usual annual soirée at the Paris Observatory in March. $\mathrm{He}$ had distributed to the leading Parisian engineering firms the conditons for the construction of the cupola for the great equatorial to be built in the newly-annexed grounds. The diameter of the revolving cupola is to be 20 metres. The form must be hemispherical. The time required for rapid revolution is 10 minutes. It is to revolve in the same direction as the heavens, and the mechanism will cause the revolution of a seat for two astronomers. From Nature 25, 249 \& 250; January 12, 1882.

determined all the evidence suggests that in theileriosis the main component of the immune response is the recognition of theilerial antigens on infected cells and the subsequent destruction of these cells by thymus derived lymphocytes.

The significance of this work is that it demonstrates the importance of cell mediated immunity in the natural host of an important protozoal disease and also extends the phenomenon of cytolytic restriction to the protozoa. Thus theileriosis joins leishmaniasis ${ }^{9}$ as examples of intracellular protozoa that are controlled by cell-mediated immune responses, class $I$ in the case of the former and class II in the latter, giving support to the suggestion that the main function of the major histocompatibility complex (MHC) molecules is to guide $T$ lymphocytes to deliver appropriate responses to diverse kinds of infection ${ }^{10}$. The diseases of man caused by Toxoplasma gondii and Trypanosoma cruzi, which are also intracellular, may soon yield up their secrets. $\square$

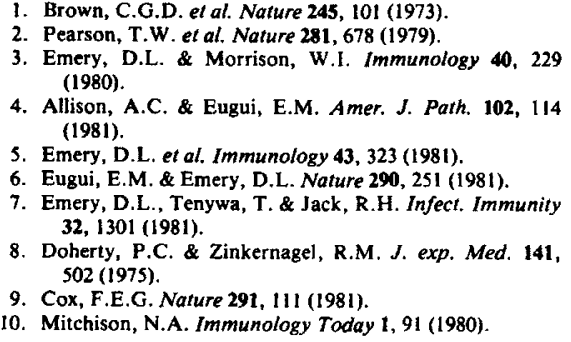

